\title{
Spray-Drying-Based Surface-Enhanced Raman Spectroscopy: Effect of the Silver Nanoparticle Aggregate Size on the Enhancement of Raman Scattering
}

Chigusa Matsumoto

Kanazawa University

Masao Gen ( $\nabla$ mgen@staff.kanazawa-u.ac.jp )

Kanazawa University

Atsushi Matsuki

Kanazawa University

Takafumi Seto

Kanazawa University

Research Article

Keywords: AgNP, RhB, Raman enhancement, AgNPs atomized, post-dropping route, hot spots

Posted Date: December 6th, 2021

DOI: https://doi.org/10.21203/rs.3.rs-1133225/v1

License: (c) (1) This work is licensed under a Creative Commons Attribution 4.0 International License.

Read Full License 


\section{Abstract}

We report a spray-drying method to fabricate silver nanoparticle (AgNP) aggregates for application in surface-enhanced Raman spectroscopy (SERS). A custom-built system was used to fabricate AgNP aggregates of three sizes, 48, 86, and $218 \mathrm{~nm}$, from drying droplets containing AgNPs atomized from an AgNP suspension. Sample solutions of Rhodamine B (RhB) at $10^{-6}, 10^{-8}$, and $10^{-10} \mathrm{M}$ concentrations were dropped onto the AgNP aggregates as probe molecules to examine the enhancement of the Raman signals of the RhB. The ordering of the analytical enhancement factors (AEFs) by aggregate size at a given RhB concentration was $86 \mathrm{~nm}>218 \mathrm{~nm}>48 \mathrm{~nm}$. The AEFs of the $86 \mathrm{~nm}$ AgNP aggregates were higher than those of the 218-nm aggregates, although the 218-nm aggregates had more hot spots where Raman enhancement occurred. This finding was attributable to the deformation and damping of the electron cloud in the highly aggregated AgNPs, reducing the sensitivity for Raman enhancement. When $\mathrm{RhB}$ was premixed with the AgNP suspension prior to atomization, the AEFs at $10^{-8} \mathrm{M}$ RhB rose $\sim 100$ fold compared to those in the earlier experiments (the post-dropping route). This significant enhancement was probably caused by the increased opportunity for the trapping of the probe molecules in the hot spots.

\section{Introduction}

Water contamination is a global concern that threatens human health ${ }^{1-4}$ and impairs the health of ecosystems $^{5-7}$. Water contaminants and pollutants include heavy metals ${ }^{8}$ and organic compounds that adversely affect human health and ecosystems ${ }^{9-12}$. Water contamination needs to be promptly detected and analyzed to sustain the environment and minimize human health hazards and ecosystem health impairments.

Heavy metal in water samples is generally detected by methods such as atomic absorption spectrometry (AAS) $)^{13,14}$, anodic stripping voltammetry (ASV) ${ }^{15}$, inductively coupled plasma mass spectrometry (ICPMS) ${ }^{16,17}$ and electrochemical detection ${ }^{18,19}$. HPLC/ICP-MS coupling detects heavy metals at rapid speeds in water even at concentrations on the order of $\mathrm{ppt}^{20}$, while the electrochemical methods are capable of detecting heavy metals onsite ${ }^{21}$. Organic pollutants, on the other hand, are detected by chromatographic and spectroscopic methods ${ }^{22,23}$. The multiple procedures required to prepare the samples for these methods introduce a risk of adulteration of the samples with impurities. Impurities may enter the samples, for example, in pretreatment steps such as the liquid-liquid extraction and solid-phase extraction ${ }^{24}$. Volatile components, moreover, are likely to be lost during the solid-phase extraction ${ }^{25}$.

Surface-enhanced Raman spectroscopy (SERS) is a facile and rapid platform for the trace analysis of organic compounds that provides a drastic enhancement of the Raman signals over traditional Raman spectroscopy. SERS stems from localized surface plasmon resonances in noble metal nanoparticles that create enormously intensified electromagnetic fields ${ }^{26}$. The significant Raman enhancement in SERS occurs at the junctions of two or more aggregated metal nanoparticles ${ }^{27-30}$, so-called hot spots. The 
degree of aggregation of the noble metal nanoparticles is a critical determinant of Raman enhancement in SERS. Hence, a number of studies have been conducted to control these aggregates ${ }^{31,32}$. The placement of probe molecules in the hot spots is also an essential step to enhance their Raman signals $^{33}$.

Aerosol techniques can be flexibly applied to many research fields, from pollution studies to the detection of biological species ${ }^{34}$ and investigations into material synthesis. One such technique, the spray-drying method, atomizes tiny droplets from stock solutions. An investigator performing the spray-drying method can control the degree of nanoparticle aggregation by varying the droplet size and the concentration of nanoparticles within the droplet ${ }^{35}$. The nanoparticle aggregate is formed as a result of solvent evaporation inside the droplets (Fig. 1). The probe molecules can also get trapped in the hot spots during aggregate formation, which may provide a mechanism for arranging the probe molecules in the hot spots more efficiently than can be done by methods using traditional SERS substrates. In the SERS substrate method, for example, the probe molecules need to diffuse into the hot spots when the analyte solution is dropped onto the SERS substrate ${ }^{36}$. An alternative approach for performing SERS has been developed using a colloidal suspension ${ }^{37,38}$. The aggregation state of the nanoparticles, however, is commonly controlled with the use of additives, that is, surfactants. The Raman signals of these additives can also be enhanced, which can potentially interfere with the signals of the probe molecules. In contrast, a spraydrying method enables the creation of nanoparticle aggregates without surfactants.

In this study we report a spray-drying method to be applied for the SERS analysis of trace amounts of probe molecules. First, we demonstrate the controllable fabrication of nanoparticle aggregates using a custom-built spray-drying system. We examine how the size of silver nanoparticle aggregates affects the SERS signals using Rhodamine B as a probe molecule. Lastly, we atomize a premixed solution of silver nanoparticles and Rhodamine $B$ to facilitate the trapping of the probe molecules in hot spots in order to further enhance their Raman signals.

\section{Methods}

Materials. Silver nanoparticles (AgNPs) were used as an SERS agent in this study. An AgNP colloidal suspension with a primary particle size of $30 \mathrm{~nm}$ (Ag Nanocolloid H-1, Mitsubishi Materials Corporation) was purchased and diluted with ultrapure water $(18.2 \mathrm{M} \Omega \mathrm{cm})$ to obtain 0.01 and $0.1 \mathrm{wt} \%$ AgNP aqueous suspensions. Rhodamine B (RhB; Kanto Chemical Co., Ltd) was chosen as a probe molecule. Briefly, 47.9 $\mathrm{mg}$ of RhB was dissolved in $10 \mathrm{~mL}$ of ultrapure water to prepare a $1.0 \times 10^{-2} \mathrm{M} \mathrm{RhB}$ stock solution. The $\mathrm{RhB}$ stock solution was further diluted with ultrapure water to obtain $1.0 \times 10^{-6}, 1.0 \times 10^{-8}$, and $1.0 \times$ $10^{-10} \mathrm{M}$ RhB solutions for the SERS experiments. All materials were used without further puri $\square$ cation.

Atomization of the AgNPs. Atomized droplets containing AgNPs were used to fabricate AgNP aggregates for SERS sensing. A schematic of the experimental setup is shown in Figure 1. The droplet atomization was carried out using a custom-built system with a pressurized two-fluid nozzle ${ }^{46}$. Briefly, a liquid sample 
was drawn into the system by the negative pressure occurring within the nozzle when a pressurized gas was introduced. A liquid film formed within the nozzle and was then broken into droplets through the force of the shear stress. An AgNP suspension at a given concentration was supplied to the system at a rate of $0.25 \mathrm{~mL} \mathrm{~min}{ }^{-1}$ using a syringe pump (70-2205, Harvard Apparatus). Ultrapure water was mixed with the AgNPs suspension before the atomization to further dilute any concentrations of potential contaminants within the suspension. $\mathrm{N}_{2}$ gas was used both as a pressurized gas and as a carrier gas to deliver the atomized droplets from the nozzle to a heating zone. $\mathrm{N}_{2}$ gas was further introduced into the heating zone at a flow rate of $4.5 \mathrm{~L} \mathrm{~min}^{-1}$, and the atomized droplets were heated to $150{ }^{\circ} \mathrm{C}$ to promote solvent evaporation and dry the AgNP aerosols. The total flow rate in the atomization system was $6.9 \mathrm{~L}$ $\mathrm{min}^{-1}$, and the droplet residence time was $0.93 \mathrm{~s}$. Finally, AgNPs in the form of dried aerosol were deposited onto a copper-made circular substrate (SEM plate Okenshoji Co.) of $14 \mathrm{~mm}$ in diameter using a custom-made impactor. The diameter of the impaction nozzle was $1 \mathrm{~mm}$, hence the AgNPs were accelerated at a flow rate of $17 \mathrm{~L} \mathrm{~min}{ }^{-1}$ for impaction. The deposited AgNPs were kept at ambient temperature and pressure for 24 hours before the SERS experiments.

AgNPs of three different aggregate sizes were fabricated, and their sensitivity in SERS analysis was examined. The smallest and largest AgNPs aggregate sizes were achieved with the 0.01 and $0.1 \mathrm{wt} . \%$ AgNP suspensions, respectively. The concentration played a role in controlling the number of AgNPs incorporated in a single droplet. In addition, AgNPs of a medium aggregate size were prepared by size selection using a differential mobility analyzer. As will be shown, aggregates of three sizes, namely, 48, 86 , and $218 \mathrm{~nm}$, were prepared for analysis in the SERS experiments.

Particle characterization. The absorption spectra of the AgNP suspensions were measured using a UV-Vis spectrometer (V-550, JASCO) to confirm the plasmon resonance of the AgNP. The scanning speed and range were $200 \mathrm{~nm} \mathrm{~min}{ }^{-1}$ and $200-800 \mathrm{~nm}$, respectively. Note that concentration of the AgNPs suspension used in the absorption measurement, $0.001 \mathrm{wt} \%$, was markedly lower than the concentration set in the experiments, as suspensions with concentrations higher than $0.001 \mathrm{wt} \%$ showed saturated absorption. (Fig. S2 in SI)

The hydrodynamic diameter of the AgNPs suspension was characterized using dynamic light scattering analysis (nano Partica SZ-100-Z, HORIBA). A $0.001 \mathrm{wt} \%$ AgNP suspension was used for the size characterizations, because multiple scattering reduced the accuracy of the measurements taken with the higher-concentration suspensions. The measurement took 120 seconds and was repeated three times. (Fig. S3)

The particle sizes and morphologies of the AgNP aerosols generated from the system were characterized using online and offline measurements. The particle size distributions were measured using a differential mobility analyzer (DMA; 3081, TSI) coupled with a condensation particle counter (CPC; 3775, TSI) as a scanning mobility particle sizer ${ }^{47}$. The DMA was also used for size selection to obtain the medium-size AgNPs aggregates. The average size of the AgNP aerosols reported in this study was the peak size of the 
particle size distribution. To calculate the average size, the size distribution was fitted with a log-normal distribution ${ }^{48-50}$.

The particle sizes and morphologies of the AgNPs deposited on the substrate were characterized with a field emission scanning electron microscope (FE-SEM; JSM-7100F, JEOL). The SEM images were taken at an accelerating voltage of $15 \mathrm{kV}$. The particle size distribution the of AgNPs deposited on the copper substrate was estimated from the Feret diameter of 100 AgNPs. The particles were deposited on the substrate over an area of about $0.785 \mathrm{~mm}^{2}$. The deposition time was $21 \mathrm{~s}, 227 \mathrm{~s}$, and $2.5 \mathrm{~s}$ for the 48-, 86-, and 218-nm AgNP aggregates, respectively.

SERS analysis. The SERS experiments were performed with AgNPs aggregates of three different sizes and $\mathrm{RhB}$. One hundred $\mu \mathrm{L}$ of RhB aqueous standard solution at a given concentration was dropped onto AgNP aggregates that had been fabricated on the copper substrate. The SERS spectra were obtained with a Raman spectrometer (Nanofinder HE, Tokyo Instruments) calibrated against a silicon wafer peak at 520 $\mathrm{cm}^{-1}$. A 532-nm laser of $0.157 \mathrm{~mW}$ was applied for an exposure time of $1 \mathrm{~s}$. More than 10 SERS spectra at different locations were obtained for each sample. The obtained spectra were analyzed with a data processing software application (Igor Pro, WaveMetrics) for background subtraction. The calculated peak area of a representative vibrational mode of $\mathrm{RhB}$ was $1649 \mathrm{~cm}^{-1}$.

\section{Results And Discussion}

Size and morphology characterization. Figure 2 shows the gas-phase size distributions of AgNP aerosols atomized from AgNP suspensions of 0.01 and $0.1 \mathrm{wt} \%$. The average size of an AgNP aerosol from the 0.01 -wt\% suspension was found to be about $38 \mathrm{~nm}$, which was roughly consistent with the size of a primary AgNP particle $(\sim 30 \mathrm{~nm})$ in the suspension. This agreement in size confirmed that a droplet generated from the $0.01 \mathrm{wt} \%$ suspension mainly contained a single AgNP. The width of the distribution was reflected by the size distribution of the droplets generated in the atomization system. The average droplet diameter was estimated to be about $1.6 \mu \mathrm{m}$, based on the size of the AgNP aerosol shown in Text $\mathrm{S} 1$ in the Supplementary Information (SI). When the suspension concentration of $0.1 \mathrm{wt} \%$ was used to generate AgNP aerosols, the average size increased to $66 \mathrm{~nm}$ (Fig. 2), more than double the size measured when using the $0.01 \mathrm{wt} \%$ suspension. On the basis of the mass balance ${ }^{39}$, the tenfold higher concentration of the suspension resulted in a threefold larger particle size at a given droplet size and given particle density, assuming that a spherical aerosol was formed from the solvent evaporation of a droplet containing colloidal particle(s). Hence, the increased average size could be attributed to the increase in the concentration of AgNPs in the droplet, that is, the suspension concentration. This size increase also suggests that a droplet generated from the $0.1 \mathrm{wt} \%$ suspension contained more than one AgNP, leading to aggregate formation. Overall, the above results demonstrate that the sizes of the aerosols generated from our system were controllable by the suspension concentration.

The SEM images in Figure 3 exhibit AgNPs deposited on the copper plates without probe molecules (i.e., RhB) added, and the corresponding size distributions of the three AgNP aggregates. AgNPs generated 
from the $0.01 \mathrm{wt} \%$ suspension formed submonolayer films (Fig. 3a). The average measured size of the primary particles was $48 \mathrm{~nm}$ (Fig. 3d), which was in fair agreement with the primary particle size (30 nm) and aerosol particle size $(38 \mathrm{~nm})$. This agreement in size suggests that the AgNPs were deposited on the substrate without much aggregate formed in the air prior to impaction. The medium aggregate size was obtained by using a 0.1-wt\% AgNP suspension to generate AgNP aerosols with a broad size range (Fig. 2) and then using DMA to extract only particles of around $70 \mathrm{~nm}$. The deposited AgNPs formed spherical aggregates (Fig. $3 \mathrm{~b}$ ) with an average measured aggregate size ( $86 \mathrm{~nm}$, Fig. 3e). Without DMA for size selection, the deposited AgNPs generated from the 0.1-wt\% suspension formed even larger spherical aggregates (Fig. 3c) with an aggregate size $(218 \mathrm{~nm}$, Fig. 3f) more than double that of the AgNP aerosols found in the gas phase measurement (Fig. 2). This size difference between the gas phase measurement and SEM observation may have been attributable to the particle bounce effect ${ }^{40}$. As discussed in the Supplementary Information (Text S3), we expected particle bounce to occur for all of the deposited particles studied. As a result, the AgNPs deposited on the substrate may have been redistributed by this expected particle bounce. Only large aggregate AgNPs, which have a higher adhesion force due to the higher contact area between a particle and a substrate ${ }^{41}$, may remain deposited. Using the two suspension concentrations and DMA, we fabricated aggregates of three different sizes: 48, 86, and 218 $\mathrm{nm}$.

Aggregate size dependence of Raman enhancement. Figure 4 shows the SERS spectra of RhB at $10^{-6}$, $10^{-8}$, and $10^{-10} \mathrm{M}$ using the aggregate sizes of 48,86 , and $218 \mathrm{~nm}$. A standard RhB solution was dropped onto the aggregates. At $10^{-6} \mathrm{M} \mathrm{RhB}$, all of the characteristic vibrational peaks were clearly observed at $500-1700 \mathrm{~cm}^{-1}$ for the AgNP aggregates of all three sizes (Fig. 4a). The peak assignments are listed in Table $1^{42}$. Among the peaks, the $\mathrm{C}-\mathrm{C}$ stretching mode at $1649 \mathrm{~cm}^{-1}$ was found to be most intense, and thus was used as the representative peak for calculating an enhancement factor. No peaks were seen in the absence of AgNPs (Fig. 4a), which confirms that the AgNP aggregates were responsible for the Raman enhancement of the RhB peaks. Raman enhancement generally increases with the number of contact points (hot spots) between SERS nanoparticles of a given primary size ${ }^{27-29}$ in the laser sensing volume. Hence, the AgNPs of the largest aggregate size in the present study, $218 \mathrm{~nm}$, were expected to show the heist peak intensity at $1649 \mathrm{~cm}^{-1}$, as they had the largest number of contact points. We were interested to find, however, that the medium-size AgNPs $(86 \mathrm{~nm})$ exhibited the highest target peak. By aggregate size, the peak intensity follows the order $86 \mathrm{~nm}>218 \mathrm{~nm}>48 \mathrm{~nm}$. The peak intensity from the 218-nm aggregates was higher than that from the 48-nm aggregates, which can be explained by the larger number of contact points. The higher peak from the $86-\mathrm{nm}$ aggregate versus that from the 218$\mathrm{nm}$ aggregate was attributable to deformation and damping of the electron cloud in the high degree of AgNP aggregation ${ }^{31,32}$. The deformation and damping effect cause redshifts of the surface plasmon excitation wavelength of the AgNPs. The redshifts reduce the excitation, and therefore the sensitivity for Raman enhancement. Similar observations have been made on the AgNP aggregates in the size range from 520 to $1600 \mathrm{~nm}^{36}$, with a higher Raman enhancement observed for the smaller aggregates. At the $10^{-8} \mathrm{M} \mathrm{RhB}$ (Fig. 4b), only the 86-nm aggregate clearly showed the RhB peaks, but no RhB peaks were 
seen for the 48 and $218 \mathrm{~nm}$ aggregates. The 48 and $218 \mathrm{~nm}$ aggregates exhibited some peaks which could not be assigned to RhB. These peaks were found to be non-reproducible and likely due to the photocarbonization $^{4344}$. No clear RhB peaks were seen in any of the aggregates at $10^{-10} \mathrm{M}$ RhB (Fig. 4c), suggesting detection limits of $>10^{-10} \mathrm{M}$ and $>10^{-8} \mathrm{M}$ RhB for the 86-nm aggregate, and the 48 and 218 $\mathrm{nm}$ aggregates, respectively, in this study.

Table 1

Main peaks of Rhodamine B and the peak assignments.

\begin{tabular}{|ll|}
\hline Raman shift $\left[\mathrm{cm}^{-1}\right]$ & Assignments \\
\hline 1199 & C-C bridge band stretching and aromatic \\
\hline 1281 & C-H bending \\
\hline 1360 & Aromatic C-C bending \\
\hline 1528 & C-H stretching \\
\hline 1649 & C-C stretching vibration mode \\
\hline
\end{tabular}

The aggregate size dependence of Raman enhancement was summarized in terms of the analytical enhancement factor (AEF) for the RhB representative peak at $\sim 1650 \mathrm{~cm}^{-1}$ (Fig. 5). The AEF was calculated with the following formula 45

$$
\mathrm{AEF}=\frac{I_{\mathrm{SERS}} / N_{\mathrm{SERS}}}{\overline{I \mathrm{RS}}_{\mathrm{R}} / N_{\mathrm{RS}}}
$$

where $I_{\text {SERS }}$ is the intensity of RhB with a given aggregate size of AgNPs in the SERS experiments, and $I_{\text {RS }}$ is the intensity of RhB without AgNPs (i.e., normal Raman measurement). $N_{\text {SERS }}$ and $N_{\text {RS }}$ are the numbers of RhB molecules for $I_{\text {SERS }}$ and $I_{R S}$, respectively, in the laser sensing volume. Note that we assume that $N_{\text {SERS }}$ equals $N_{\text {RS }}$ in this study, as the laser sensing volume is the same in the SERS and normal Raman measurements. The SERS experiments for $10^{-6} \mathrm{M}$ RhB gave AEF values of $2.4 \times 10^{3}, 4.2 \times 10^{3}$, and $4.0 \times$ $10^{3}$ for the 48-, 86-, and 218-nm AgNP aggregates, respectively, whereas the AEF value at $10^{-8} \mathrm{M} \mathrm{RhB}$ available only for the $86-\mathrm{nm}$ aggregate was $3.4 \times 10^{4}$. These results demonstrate that the $86-\mathrm{nm}$ AgNP aggregate was the most sensitive nanostructure in the present study. The high sensitivity attained can be attributed to the optimal AgNP aggregate size, as discussed above.

Premixed atomization of AgNPs and probe molecules. Figures 4 and 5 demonstrate that the $86 \mathrm{~nm} \mathrm{AgNP}$ aggregate was the most sensitive nanostructure in the SERS sensing. Here, we further explored Raman enhancement in the 86-nm AgNP aggregates by premixing RhB with the AgNP suspension before the atomization (i.e., by taking the premixed atomization route). Figure 6 shows SERS spectra of RhB at $10^{-6}$, 
$10^{-8}$, and $10^{-10} \mathrm{M}$ concentrations in the 86-nm AgNP aggregates. Similar to the earlier experiments, no clear RhB peaks were seen at $10^{-10} \mathrm{M}$. The AEF values at $10^{-6}$ and $10^{-8} \mathrm{M}$ RhB were found to be higher with the premixed atomization than with the post-dropping (Fig. 7). The AEF values with premixed atomization were estimated to be $5.1 \times 10^{4}$ and $3.7 \times 10^{6}$ for the $10^{-6}$ and $10^{-8} \mathrm{M}$ concentrations, respectively, or 12 and 110 times higher than the AEF values with the post-dropping. The further Raman enhancement in the premixed atomization route was attributable to the greater number of opportunities for the trapping of the probe molecules (RhB) in hot spots (Fig. 8). The increased trapping of RhB in hot spots increased AEF values.

\section{Conclusions}

We have reported a spray-drying method to fabricate highly sensitive SERS platforms based on AgNP aerosol aggregates. AgNP aggregates of three sizes, 48, 86, and $218 \mathrm{~nm}$, were prepared using a custombuilt system. The AEFs of the 48-, 86-, and 218-nm AgNP aggregates treated by the post-dropped $10^{-6} \mathrm{M}$ RhB were $2.4 \times 10^{3}, 4.2 \times 10^{3}$, and $4.0 \times 10^{3}$, respectively, whereas that treated by the post-dropped $10^{-8}$ $\mathrm{M} \mathrm{RhB}$, which could be detected for only the $86-\mathrm{nm}$ aggregate, was $3.4 \times 10^{4}$. We found that the $86-\mathrm{nm}$ AgNP aggregate was the most sensitive nanostructure in the present study. The higher Raman enhancement of the 86-nm aggregate versus that of the 218-nm aggregate was attributable to the deformation and damping of the electron cloud in the highly aggregated AgNPs, a phenomenon that reduced the sensitivity for Raman enhancement.

When RhB was premixed with the AgNP suspension before atomization, the Raman enhancement was greatly enhanced compared to that in the earlier experiments adopting the post-dropping route. The AEFs of the 86-nm aggregates formed by the premixed atomization route were $5.1 \times 10^{4}$ and $3.7 \times 10^{6}$ at the $10^{-6}$ and $10^{-8} \mathrm{M} \mathrm{RhB}$ concentrations, respectively. These AEFs were 12 and 110 times higher than those formed by the post-dropping route. The greater Raman enhancement in the premixed atomization route can be ascribed to the greater number of probe molecules (RhB) to be trapped in the hot spots (Fig. 8) versus that under the premixed atomization condition. This route is expected to be a promising method for the trace analysis of environmental pollutants.

\section{Declarations}

\section{Acknowledgements}

The authors gratefully acknowledge support from JST CREST Grant Number JPMJCR18H4, Japan, KAKENHI 21H01687, and the Japan Science and Technology Agency (JST) for Fusion Oriented Research for disruptive Science and Technology program (JPMJFR206V).

\section{Author contributions}


C.M.: study design; acquisition, analysis, and interpretation of the data; preparation of the graphs and manuscript.

M.G.: supervision; acquisition, analysis, and interpretation of the data; editing of the manuscript.

A.M.: device adjustment; acquisition and analysis of the data.

T.S.: study design and supervision; interpretation of the data.

All of the authors have read and approved this manuscript.

\section{Additional information}

Competing interests The authors declare no competing interests.

\section{References}

1. Albanese, S., Taiani, M. V. E., De Vivo, B. \& Lima, A. An environmental epidemiological study based on the stream sediment geochemistry of the Salerno province (Campania region, Southern Italy). J. Geochemical Explor, 131, 59-66 (2013).

2. Li, Z., Ma, Z., van der Kuijp, T. J., Yuan, Z. \& Huang, L. A review of soil heavy metal pollution from mines in China: Pollution and health risk assessment. Sci. Total Environ, 468-469, 843-853 (2014).

3. Mazumdar, K. \& Das, S. Phytoremediation of pb, zn, fe, and mg with 25 wetland plant species from a paper mill contaminated site in north east india. Environ. Sci. Pollut. Res, 22, 701-710 (2015).

4. Kumar, V. et al. Assessment of heavy-metal pollution in three different Indian water bodies by combination of multivariate analysis and water pollution indices. Hum. Ecol. Risk Assess, 26, 1-16 (2020).

5. Soliman Sabra, F. \& El-Deeb Mehana, E. S. Pesticides Toxicity in Fish with Particular Reference to Insecticides. Asian J. Agric. Food Sci, 3, 2321-1571 (2015).

6. Baruš, V., Jarkovský, J. \& Prokeš, M. Philometra ovata (Nematoda: Philometroidea): A potential sentinel species of heavy metal accumulation. Parasitol. Res, 100, 929-933 (2007).

7. Voogt, P., De \& KC Jones \& Persistent organic pollutants (POPs): state of the science. Environ. Pollut, 100, 209-221 (1999).

8. Fernández-Luqueño, F. et al. Heavy metal pollution in drinking water - a global risk for human health: A review. African J. Environ. Sci. Technol, 7, 567-584 (2013).

9. Huang, S., Shao, G., Wang, L., Wang, L. \& Tang, L. Distribution and health risk assessment of trace metals in soils in the golden triangle of Southern Fujian province, China.Int. J. Environ. Res. Public Health16, (2019)

10. Magge, H., Sprinz, P., Adams, W. G., Drainoni, M. L. \& Meyers, A. Zinc protoporphyrin and Iron deficiency screening: Trends and therapeutic response in an Urban pediatric center. JAMA Pediatr, 167, 361-367 (2013). 
11. Wu, B., Zhang, Y., Zhang, X. \& Cheng, S. Health risk from exposure of organic pollutants through drinking water consumption in Nanjing, China. Bull. Environ. Contam. Toxicol, 84, 46-50 (2010).

12. Rocher, V., Siaugue, J. M., Cabuil, V. \& Bee, A. Removal of organic dyes by magnetic alginate beads. Water Res, 42, 1290-1298 (2008).

13. Cabrera, C., Lorenzo, M. L. \& Lopez, M. C. Electrothermal atomic absorption spectrometric determination of cadmium, copper, iron, lead, and selenium in fruit slurry: analytical application to nutritional and toxicological quality control. J. AOAC Int, https://doi.org/doi:10.1093/jaoac/78.4.1061. (1995).

14. Huang, S., Da \& Shih, K. Y. Direct determination of zinc in seawater by graphite furnace atomic absorption spectrometry. Spectrochim. Acta Part B At. Spectrosc, 50, 837-846 (1995).

15. Olsen, K. B., Wang, J., Setiadji, R. \& Lu, J. Field Screening of Chromium, Cadmium, Zinc, Copper, and Lead in Sediments by Stripping Analysis. Environ. Sci. Technol, 28, 2074-2079 (1994).

16. Chen, $X$. et al. Rapid speciation analysis of mercury in seawater and marine fish by cation exchange chromatography hyphenated with inductively coupled plasma mass spectrometry. J. Chromatogr. A, 1314, 86-93 (2013).

17. Packer, A. P., Giné, M. F. \& Miranda, C. E. S. Dos Reis, B. F. Automated on-line preconcentration for trace metals determination in water samples by inductively coupled plasma mass spectrometry. $J$. Anal. At. Spectrom, 12, 563-566 (1997).

18. Priya, T., Dhanalakshmi, N., Thennarasu, S. \& Thinakaran, N. A novel voltammetric sensor for the simultaneous detection of $\mathrm{Cd} 2+$ and $\mathrm{Pb} 2+$ using graphene oxide/ $\mathrm{k}$-carrageenan/L-cysteine nanocomposite. Carbohydr. Polym, 182, 199-206 (2018).

19. Zhang, Y. et al. A generalized in situ electrodeposition of Zn doped CdS-based photoelectrochemical strategy for the detection of two metal ions on the same sensing platform. Biosens. Bioelectron, 77, 936-941 (2016).

20. Vogl, J. \& Heumann, K. G. Determination of heavy metal complexes with humic substances by HPLC/ICP-MS coupling using on-line isotope dilution technique. Fresenius. J. Anal. Chem, 359, 438441 (1997).

21. Wu, Z. et al. Mesoporous MgO nanosheets: 1,6-hexanediamin-assisted synthesis and their applications on electrochemical detection of toxic metal ions. J. Phys. Chem. Solids, 74, 1032-1038 (2013).

22. Koeber, R., Bayona, J. M. \& Niessner, R. Determination of benzo[a]pyrene diones in air particulate matter with liquid chromatography mass spectrometry. Environ. Sci. Technol, 33, 1552-1558 (1999).

23. Li, C. W., Benjamin, M. M. \& Korshin, G. V. Use of UV spectroscopy to characterize the reaction between NOM and free chlorine. Environ. Sci. Technol, 34, 2570-2575 (2000).

24. Hoenig, M. Preparation steps in environmental trace element analysis - Facts and traps., 54, 10211038 (2001).

25. Muir, D. \& Sverko, E. Analytical methods for PCBs and organochlorine pesticides in environmental monitoring and surveillance: A critical appraisal. Anal. Bioanal. Chem, 386, 769-789 (2006). 
26. Jeanmaire, D. L. \& Duyne, R. P. VAN. Surface Raman Spectroelectrochemistry Part1. Heterocyclic. J. Electroanal Chem, 84, 1 (1977).

27. Nie, S. \& Emory, S. R. Probing single molecules and single nanoparticles by surface-enhanced Raman scattering. Science (80-), 275, 1102-1106 (1997).

28. Pieczonka, N. P. W. \& Aroca, R. F. Single molecule analysis by surfaced-enhanced Raman scattering. Chem. Soc. Rev, 37, 946-954 (2008).

29. Liang, H., Li, Z. P., Wang, W., Wu, Y. \& Xu, H. Highly surface-roughened 'flower-like' silver nanoparticles for extremely sensitive substrates of surface-enhanced Raman scattering. Adv. Mater, 21, 46144618 (2009).

30. Sun, Y. et al. Highly sensitive surface-enhanced raman scattering substrate made from superaligned carbon nanotubes. Nano Lett, 10, 1747-1753 (2010).

31. Wokaun, A., Gordon, J. P. \& Liao, P. F. Radiation damping in surface-enhanced Raman scattering. Phys. Rev. Lett, 48, 957-960 (1982).

32. Meier, M. \& Wokaun, A. Enhanced fields on large metal particles: dynamic depolarization. Opt. Lett, https://doi.org/doi:10.1364/ol.8.000581. (1983).

33. Xu, H., Aizpurua, J., Käll, M. \& Apell, P. Electromagnetic contributions to single-molecule sensitivity in surface-enhanced Raman scattering. Phys. Rev. E -Stat. Physics, Plasmas, Fluids, Relat. Interdiscip. Top, 62, 4318-4324 (2000).

34. Sebastian Schlücker. SERS microscopy: nanoparticle probes and biomedical applications. Chemphyscem, 10, 1344-1354 (2009).

35. Iskandar, F., Lenggoro, I. W., Xia, B. \& Okuyama, K. Functional nanostructured silica powders derived from colloidal suspensions by sol spraying. J. Nanoparticle Res, 3, 263-270 (2001).

36. Kahraman, M., Aydin, Ã. \& Çulha, M. Size effect of 3D aggregates assembled from silver nanoparticles on surface-enhanced Raman scattering. ChemPhysChem, 10, 537-542 (2009).

37. Sun, L. et al. Controllable synthesis of silver nanoparticle aggregates for surface-enhanced raman scattering studies. J. Phys. Chem. C, 115, 16295-16304 (2011).

38. Zhang, Z. \& Zhu, W. Controllable-density nanojunctions as SERS substrates for highly sensitive detection. Appl. Surf. Sci, 333, 214-219 (2015).

39. Wang, W. N., Widiyastuti, W., Wuled Lenggoro, I., Kim, O., Okuyama, K. \& T. \& Photoluminescence Optimization of Luminescent Nanocomposites Fabricated by Spray Pyrolysis of a Colloid-Solution Precursor. J. Electrochem. Soc, 154, J121 (2007).

40. Cheng, Y. S. \& Yeh, H. C. Particle Bounce in Cascade Impactors. Environ. Sci. Technol, 13, 1392-1396 (1979).

41. Uryukov, B. A. \& Tkachenko, G. V. Adhesion of a plastic particle to a substrate upon high-speed collision: A theoretical model. Powder Metall. Met. Ceram, 48, 169-181 (2009).

42. Moreno, V., Murtada, K., Zougagh, M. \& Ríos, Ã. Analytical control of Rhodamine B by SERS using reduced graphene decorated with copper selenide. Spectrochim. Acta-Part A Mol. Biomol. Spectrosc, 
223, 117302 (2019).

43. Cooney, R. P., Mahoney, M. R. \& Howard, M. W. Intense raman spectra of surface carbon and hydrocarbons on silver electrodes. Chem. Phys. Lett, 76, 448-452 (1980).

44. Suzuki, S. \& Yoshimura, M. Chemical Stability of Graphene Coated Silver Substrates for SurfaceEnhanced Raman Scattering. Sci. Rep, 7, 1-7 (2017).

45. Le Ru, E. C., Blackie, E., Meyer, M. \& Etchegoint, P. G. Surface enhanced raman scattering enhancement factors: A comprehensive study. J. Phys. Chem. C, 111, 13794-13803 (2007).

46. Ichihara, F., Lee, K., Sakamoto, M., Higashi, H. \& Seto, T. Aerosolization of colloidal nanoparticles by a residual-free atomizer. Aerosol Sci. Technol, 54, 1223-1230 (2020).

47. Wang, S. C. \& Flagan, R. C. Scanning electrical mobility spectrometer. Aerosol Sci. Technol, 13, $230-$ 240 (1990).

48. Heintzenberg, J. Properties of the log-normal particle size distribution. Aerosol Sci. Technol, 21, 4648 (1994).

49. Al-Thyabat, S. \& Miles, N. J. An improved estimation of size distribution from particle profile measurements. Powder Technol, 166, 152-160 (2006).

50. Thomas, J. C. The Determination of Log Normal Particle Size Distributions by Dynamic Light Scattering. J. Colloid Interface Sci, 117, 187-192 (1987).

\section{Figures}

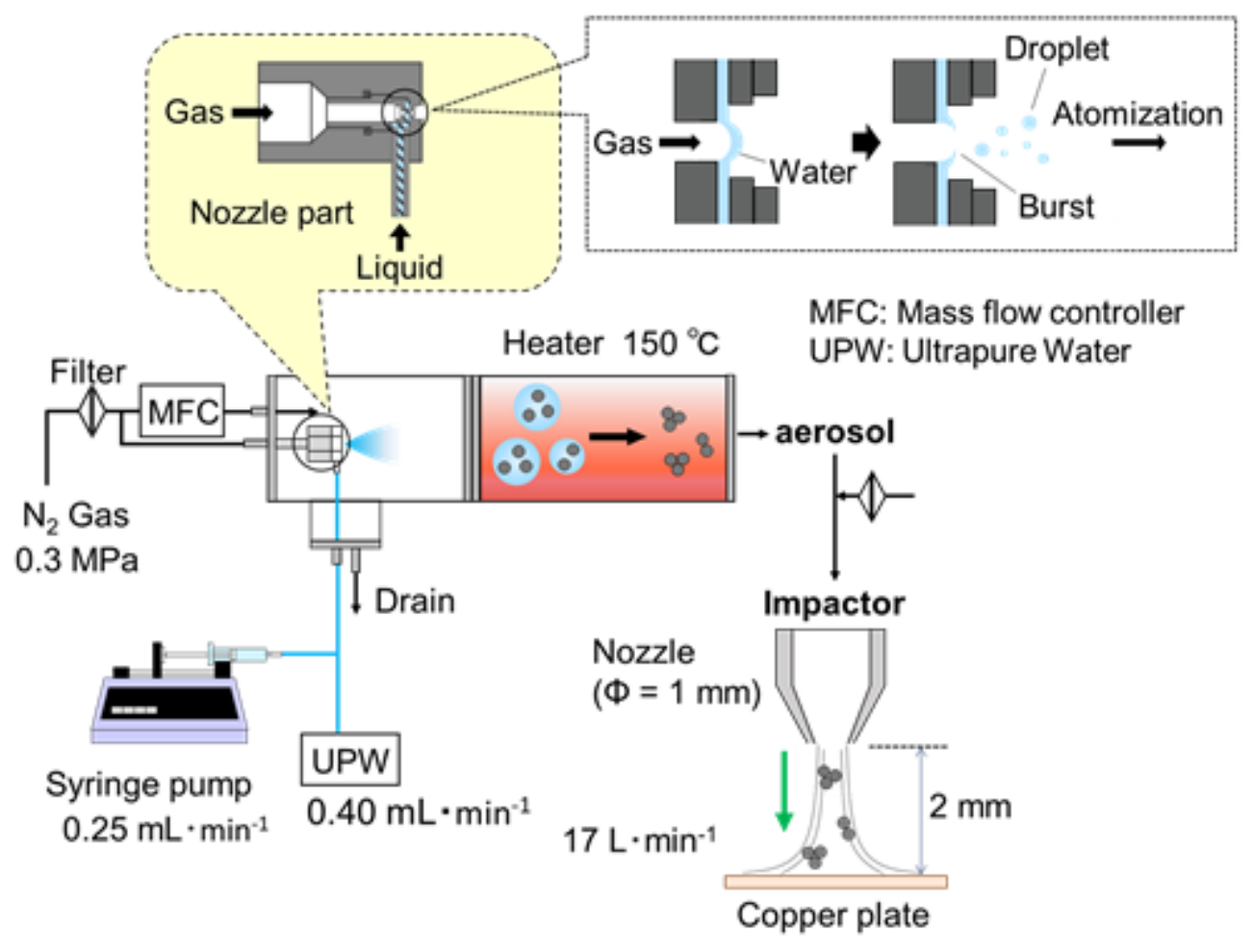

Figure 1 
Schematic of an atomization system for the fabrication of aggregated silver nanoparticles.

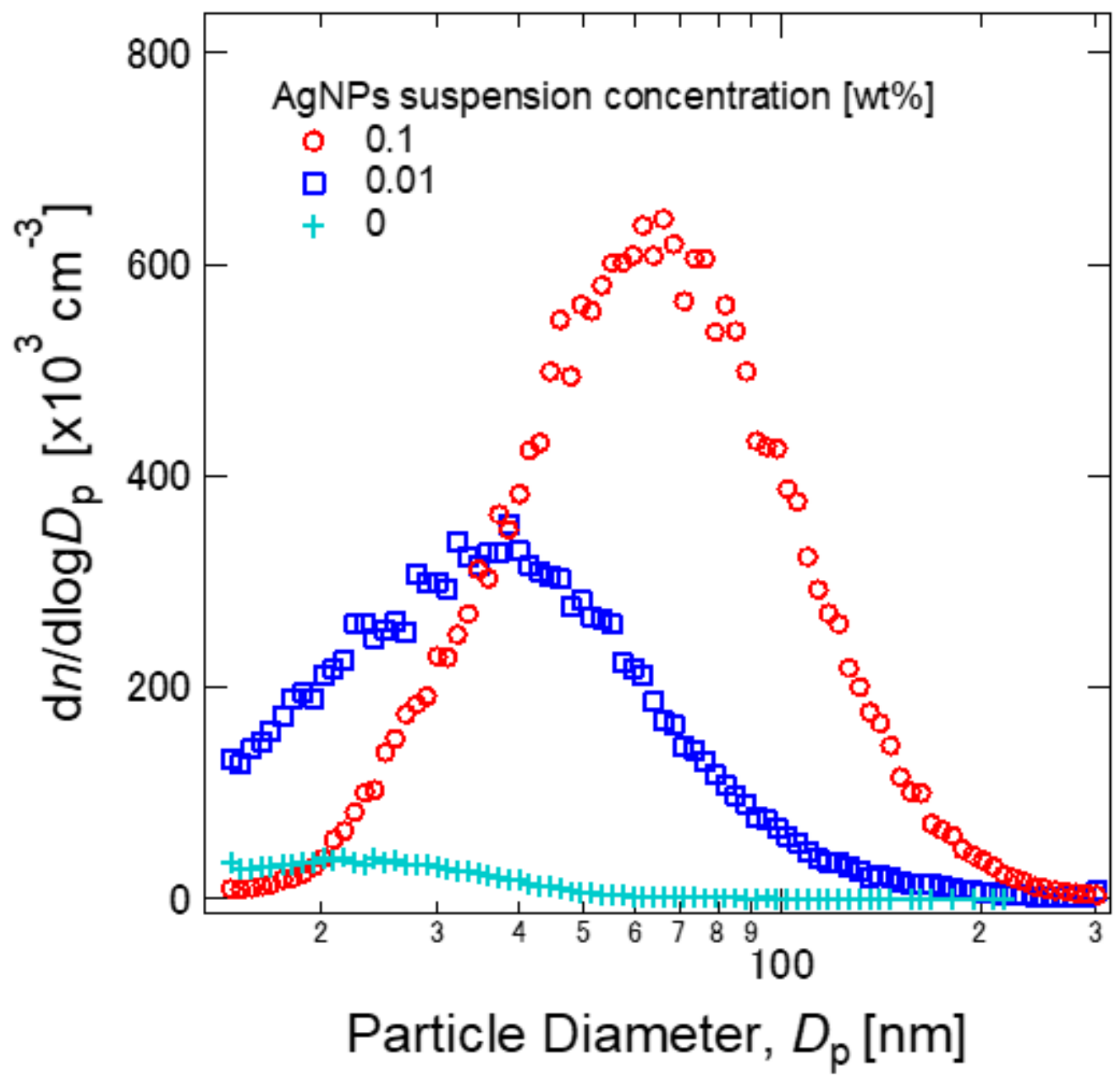

Figure 2

Size distributions of the AgNPs generated from the $0.01-, 0.1-$, and $0-w t \%$ sample suspensions. Note that the $0 \mathrm{wt} \%$ condition used only water for the atomization. 

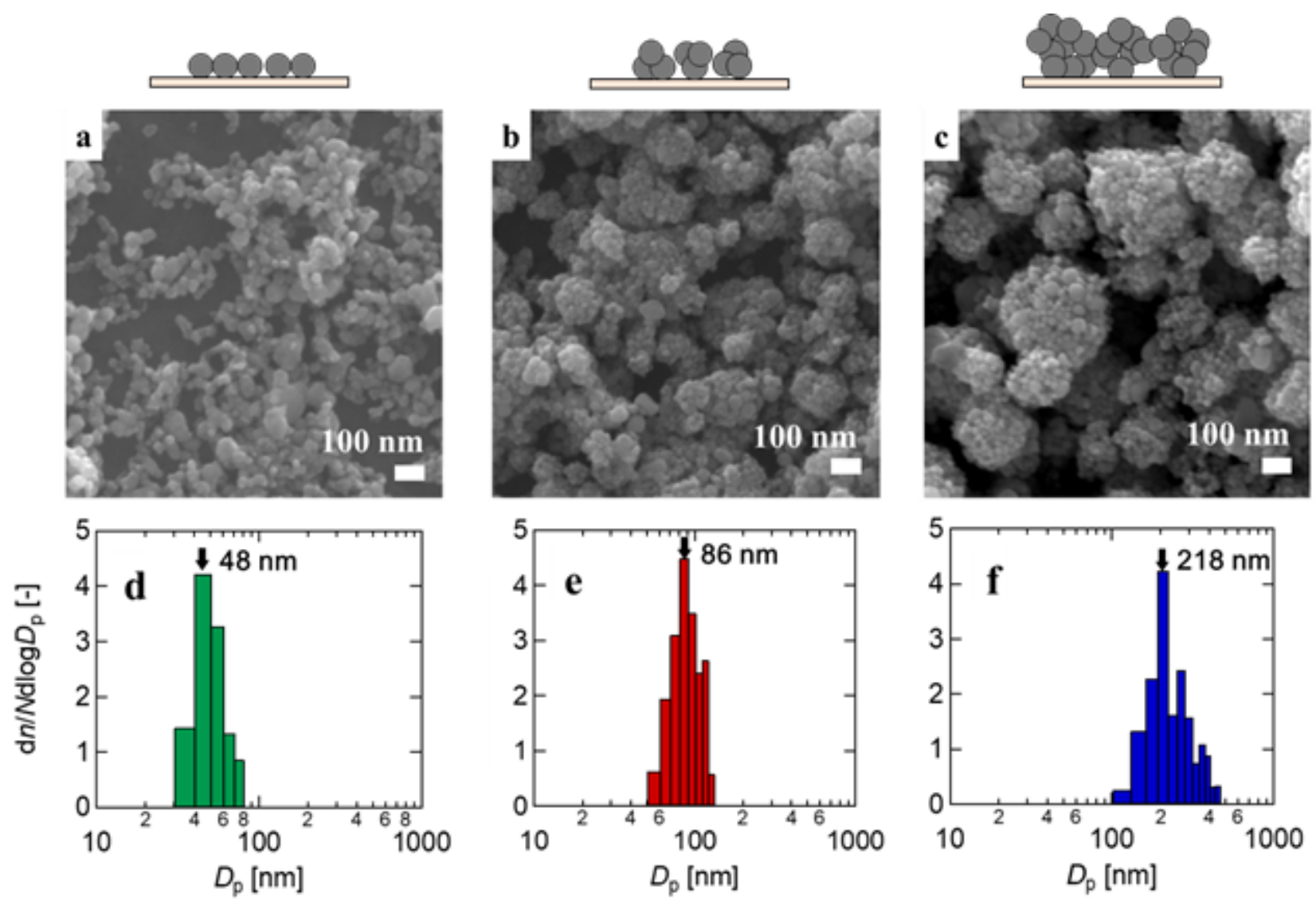

Figure 3

SEM images and size distributions of the deposited AgNPs. (a, d) $48 \mathrm{~nm}$, (b, e) $86 \mathrm{~nm}$, and (c, f) $218 \mathrm{~nm}$. 

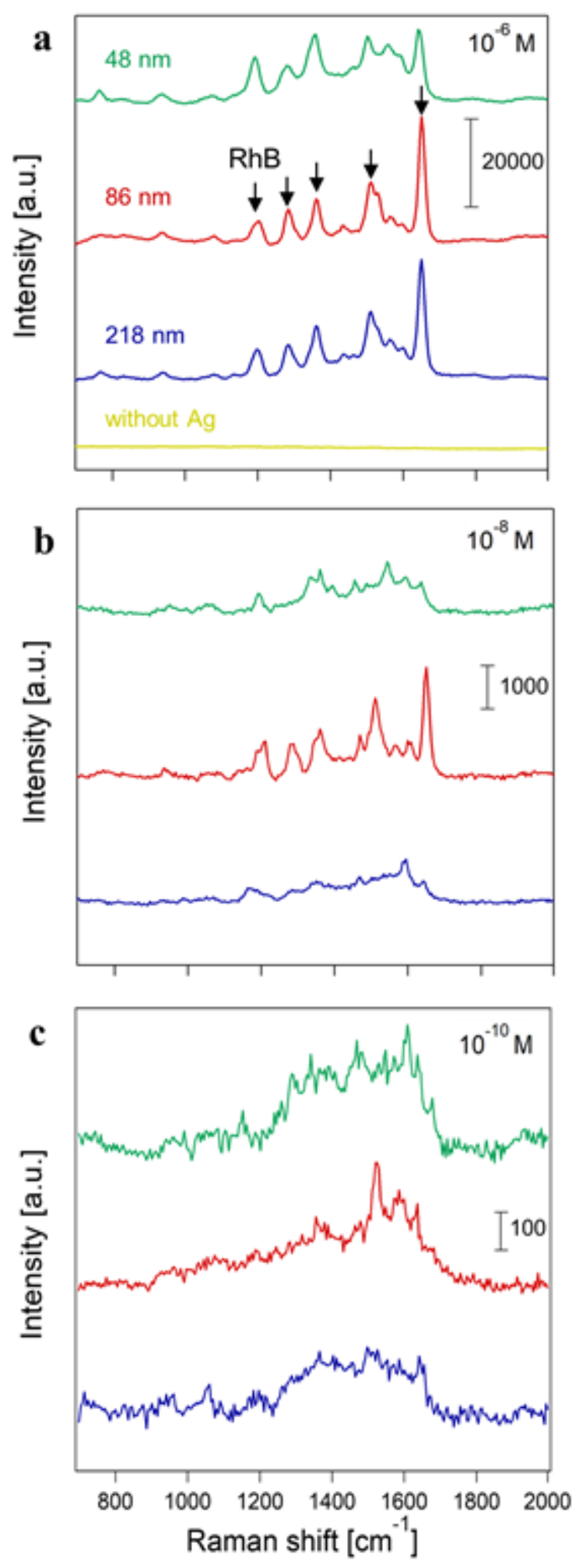

\section{Figure 4}

SERS spectra of RhB at the (a) 10-6 M, (b) 10-8 M, and (c) 10-10 M concentrations. The arrows indicate the representative $\mathrm{RhB}$ peaks (Table 1 ). 


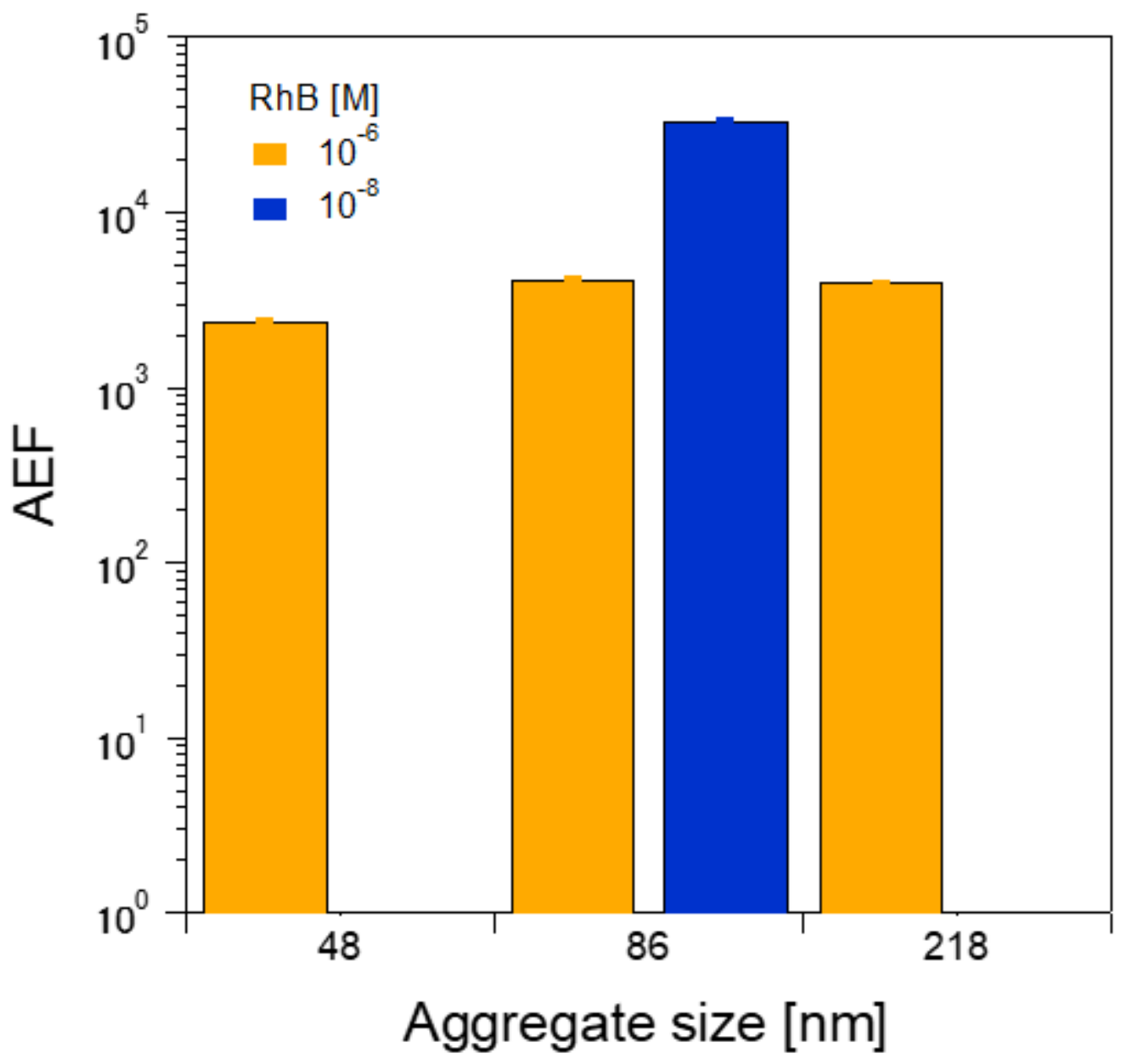

Figure 5

AEF values at 10-6 and 10-8 M RhB concentrations for the AgNP aggregate sizes of 48,86 , and $218 \mathrm{~nm}$. No AEF values were calculated the 48- and 218-nm aggregate at the 10-8 M RhB concentrations because of the absence of RhB peaks. 


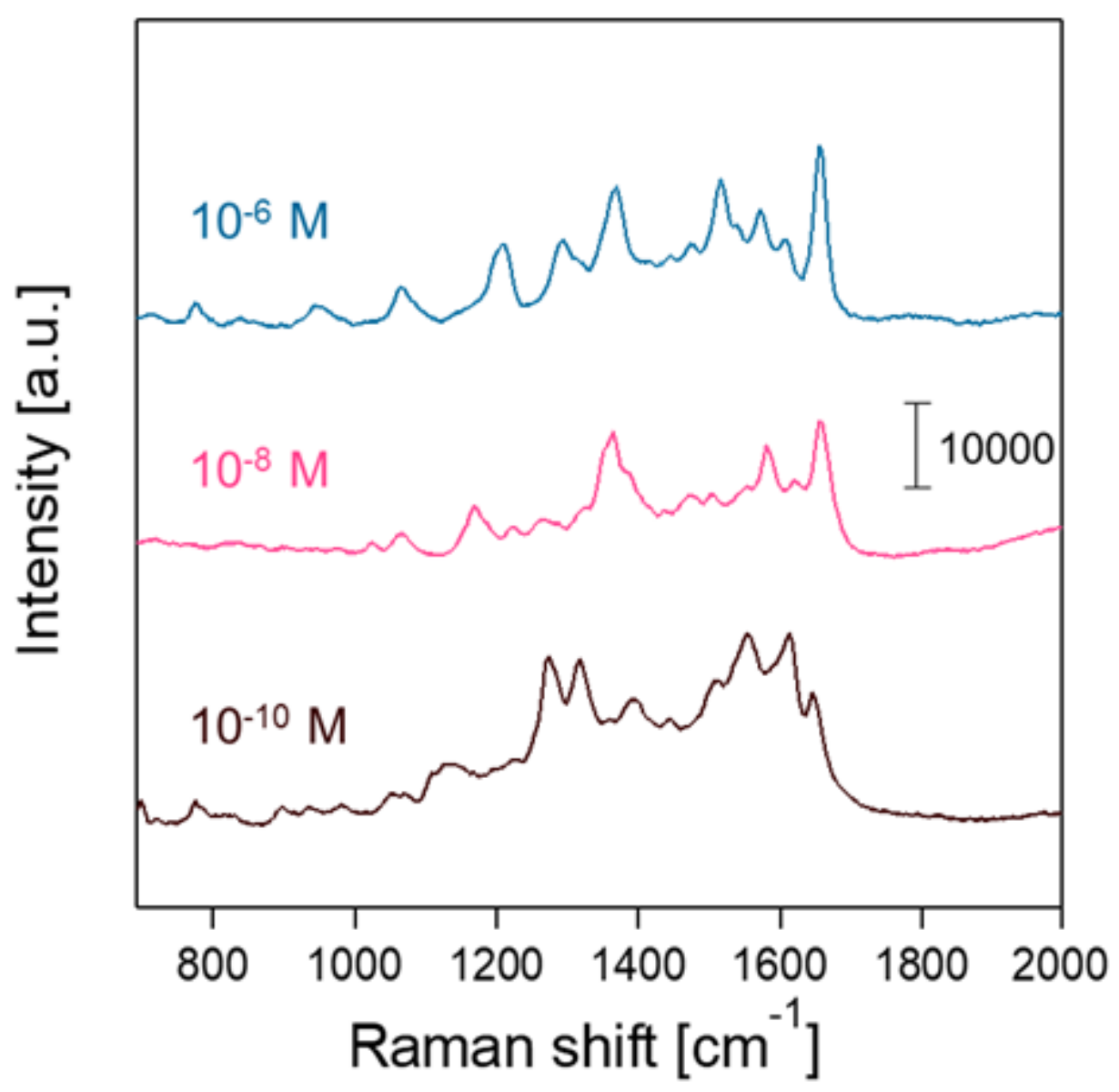

Figure 6

SERS spectra of the $86 \mathrm{~nm}$ AgNP aggregates with (a) 10-6 M, (b) 10-8 M, and (c) 10-10 M RhB in the premixed atomization route. 


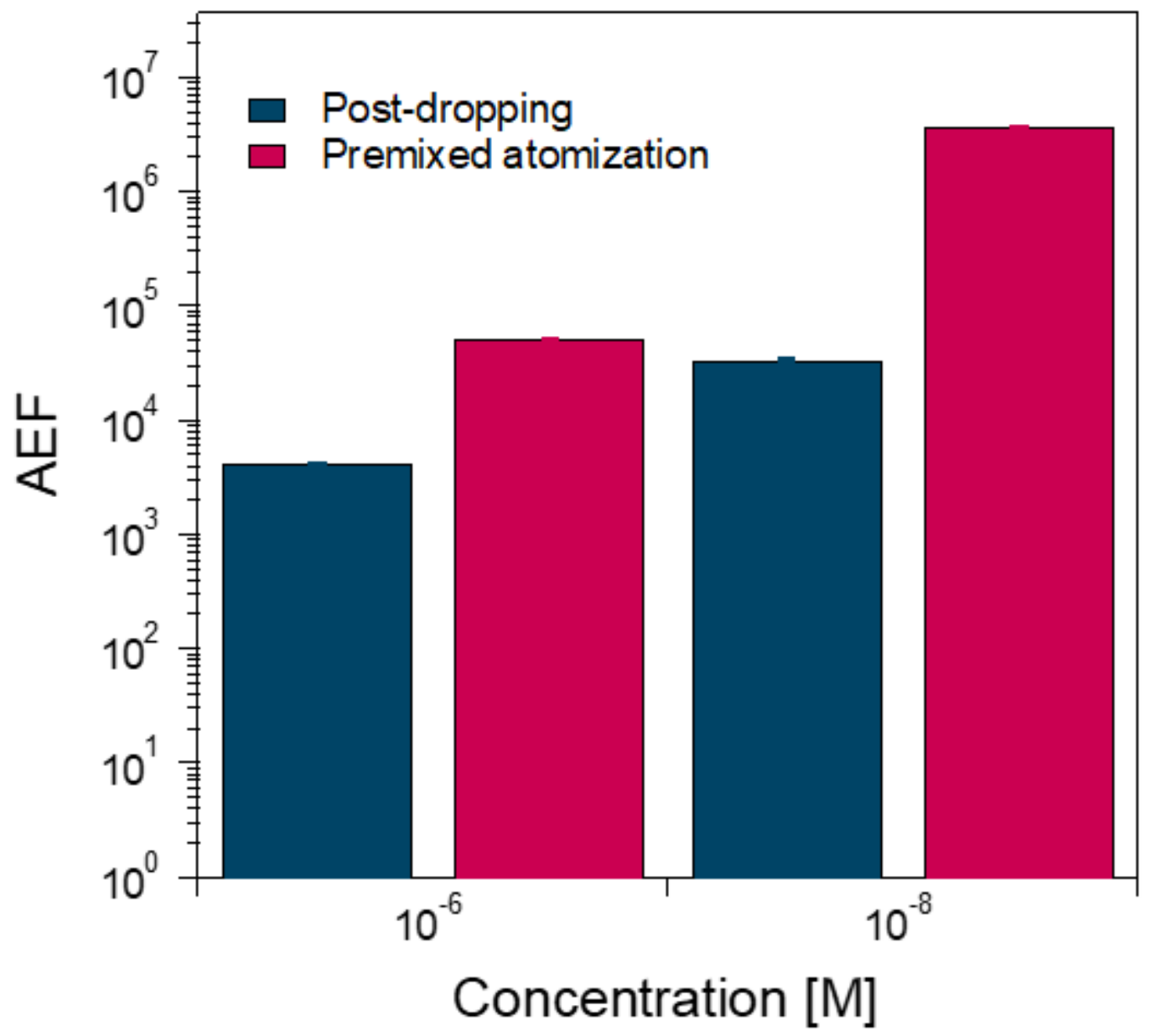

Figure 7

AEF values obtained from the $86 \mathrm{~nm}$ AgNP aggregates in the post-dropping and premixed atomization routes. 
(a) Post-dropping

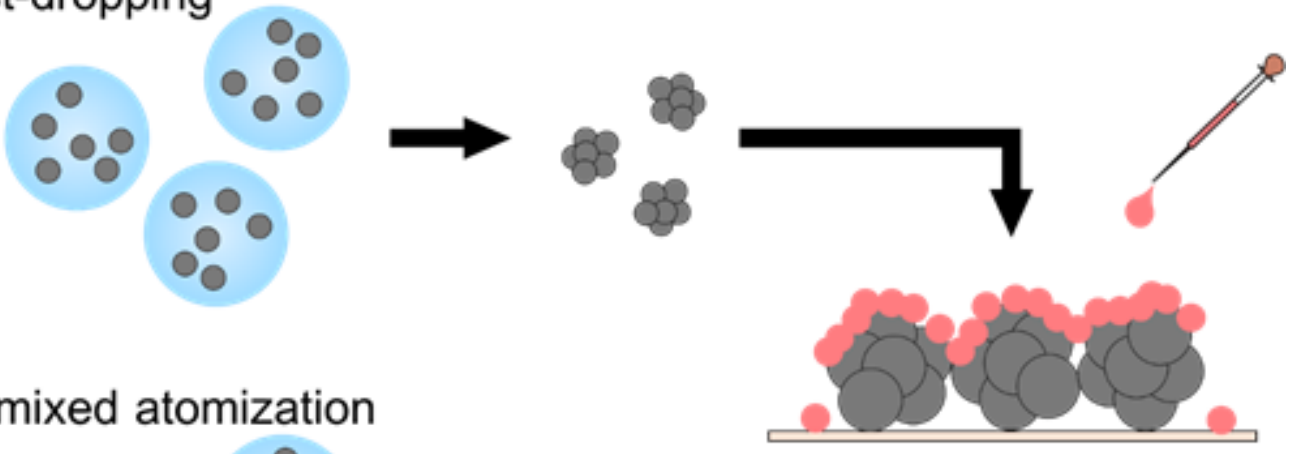

(b) Premixed atomization
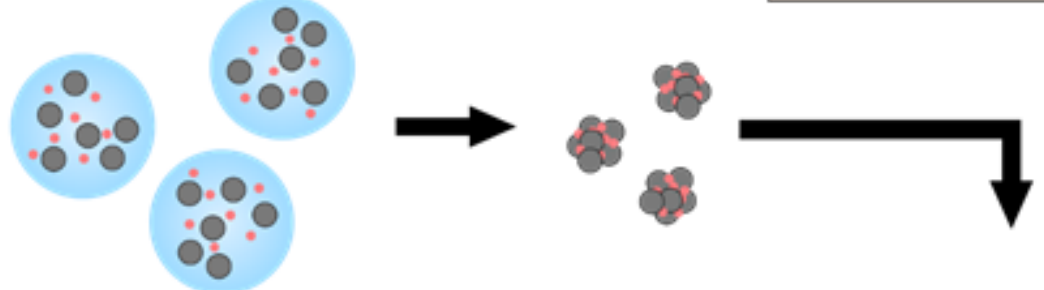

\section{AgNPs}

RhB

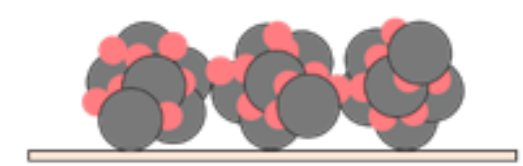

Figure 8

(a) Post-dropping and (b) premixed atomization routes for comparison of the hot spots they create.

\section{Supplementary Files}

This is a list of supplementary files associated with this preprint. Click to download.

- SIMG.pdf 